\title{
Relação entre o tempo de clampeamento do cordão umbilical e incidência de Icterícia Neonatal e níveis de hematócrito em recém-nascidos a termo saudáveis
}

\author{
Relationship between umbilical cord clamping time and incidence of Neonatal Jaundice and \\ hematocrit levels in healthy term newborns
}

\author{
Narcizo Leopoldo Eduardo da Cunha Sobieray ${ }^{1}$, Izabelle Schermak das Neves ${ }^{2}$, Thayná Skrobot ${ }^{2}$
}

\section{Resumo}

Objetivo: o objetivo deste estudo foi analisar a relação entre o tempo de clampeamento do cordão umbilical e incidência de Icterícia Neonatal e níveis de hematócrito em RN a termo saudáveis em uma maternidade de baixo risco em Curitiba de abril de 2015 a março de 2016. Métodos: Foram analisados prontuários de $188 \mathrm{RN}$, obtendo informações como via de parto, tempo de clampeamento e descrição de icterícia nos $R N$. Em 92 destes, foram coletadas amostras de sangue por capilaridade para avaliação dos níveis de hematócrito. Estabeleceu-se como clampeamento precoce aquele realizado antes dos 60 segundos e tardio os realizados em um tempo maior ou igual a 60 segundos. Resultados: Observou-se icterícia em 25\% dos RN. A média do tempo de clampeamento do grupo sem icterícia foi 105 segundos em comparação com 108 segundos no grupo com icterícia, sem associação significante $(p=0,068)$ $O$ risco relativo de um $R N$ clampeado tardiamente apresentar icterícia foi 2,18 vezes maior do que em um $R N$ clampeado precocemente ( $R R=2,18$ IC 95\% 0,15-1,71). Quanto aos níveis de hematócrito realizados, no grupo de clampeamento tardio o valor médio foi de $58,05 \%$ e 09 destes apresentaram valores $>65,0 \%$. No grupo de clampeamento precoce não houve níveis de hematócrito > 65\%. Conclusão: A média do tempo de clampeamento dos grupos foi bastante similar, o que não nos permitiu concluir que RN com maior tempo

1. Professor Adjunto do Departamento de Tocoginecologia da Universidade Federal do Paraná, Complexo Hospital de Clínicas. Curitiba-PR - Brasil

2. Acadêmicas do Curso de Medicina da Universidade Federal do Paraná. Curitiba-PR - Brasil

Endereço para correspondência: Narcizo Leopoldo Eduardo da Cunha Sobieray. Departamento de Tocoginecologia, Complexo Hospital de Clinicas da UFPR. Rua General Carneiro, 181 - Centro-82590-300 - Curitiba-PR - Brasil. E-mail: narsobieray@ gmail.com

Os autores declaram não haver conflito de interesse.

Número do protocolo de aprovação do trabalho pelo Comitê de Ética em Pesquisa/CEP-HC: $n^{\circ}$ 43241115.0.0000.0096.

Pesquisa financiada com recursos próprios. de clampeamento apresentaram mais icterícia. No entanto, o risco relativo foi maior para esta situação. Os niveis de hematócrito foram maiores no grupo com clampeamento tardio. Outros estudos são necessários para se avaliar o impacto na prevenção de Anemia no lactente.

Descritores: Cordão umbilical, Icterícia neonatal, Anemia, Hiperbilirrubinemia, Recém-nascido

\begin{abstract}
Purpose: The objective of this study was to analyze the relationship between umbilical cord clamping time and incidence of Neonatal Jaundice and hematocrit levels in healthy term newborns (NB) in a low-risk maternity hospital in Curitiba, from April of 2015 to march of 2016. Methods: We analyzed charts of 188 NB, obtaining information such as the way of delivery, clamping time and description of jaundice in NB. In 92 of these, capillary blood samples were collected for evaluation of hematocrit levels. It was established as an early clamping that performed before 60 seconds and late that performed in a time greater than or equal to 60 seconds. Results: Jaundice was observed in 25\% of NB. The mean clamping time of the non-jaundiced group was 105 seconds compared to 108 seconds in the jaundiced group., without significant association $(p=0,068)$. The relative risk of a late-clamped $N B$ presenting jaundice was 2.18 times greater than in an early clamping $(R R=2.18$, 95\% CI, 0.15-1.71). As for hematocrit levels, the mean value of late clamping group was $58.05 \%$ and 09 of these presented values $>65.0 \%$. In the early clamping group there were no levels of hematocrit $>65 \%$.Conclusion: The mean clamping time of the groups was quite similar, which did not allow us to conclude that NB with a longer clamping time had more jaundice. However, the relative risk was increased for this situation. Hematocrit levels were higher in the late clamping group. Other studies are necessary to evaluate the impact on the prevention of anemia in the infant.
\end{abstract}

Keywords: Umbilical cord; Neonatal jaundice; Anemia; Hyperbilirubinemia; Infant, newborn 


\section{Introdução}

O clampeamento do cordão umbilical é uma prática recorrente na rotina obstétrica, faz parte do manejo ativo da terceira fase do trabalho de parto e tem sido constantemente debatido na sociedade científica nos últimos anos. Conhecidamente motivo de controvérsia, o tempo de clampeamento do cordão umbilical é discutido desde séculos atrás, podendo ser encontrado relatos de publicações desde 1801, as quais abordam tal conduta $^{(1)}$. Hoje sabe-se que as relações materno-fetais e as trocas oriundas da conexão placentária entre mãe e feto são de extrema relevância, não apenas para uma gestação saudável, mas também para a vida extrauterina, especialmente nos primeiros seis meses de vida ${ }^{(1-2)}$.

Segundo a Organização Mundial da Saúde (OMS) (2), considera-se clampeamento precoce todo aquele realizado até os 60 primeiros segundos após o nascimento, sendo considerado tardio aquele realizado após o primeiro minuto de nascimento ou quando a pulsação do cordão umbilical cessa. A OMS recomenda que seja realizado o clampeamento tardio, com tempo de espera entre 1 a 3 minutos antes do clampeamento. O clampeamento precoce é apenas recomendado em casos de necessidade de reanimação imediata do neonato, sendo geralmente necessária a transferência deste para outro local adequado do Serviço de Neonatologia. Uma das principais questões relacionadas ao tempo de clampeamento é em relação ao nível de hemoglobina, hematócrito e estoque de ferro ${ }^{(3)}$. Hoje, acredita-se que o clampeamento tardio seja benéfico para o neonato quando se trata desses aspectos ${ }^{(4)}$. Um dos maiores empecilhos para a prática de clampeamento tardio seria o receio dos obstetras e pediatras, principalmente em relação às consequências desta prática, como possível hiperbilirrubinemia e policitemia neonatais ${ }^{(5)}$.

O clampeamento precoce foi por muito tempo consagrado por ter sido considerado redutor do risco de hemorragia pós-parto. Contudo, em uma recente revisão sistemática ${ }^{(6)}$ foi demonstrado que não há diferença significativa em índices de hemorragia pós-parto em clampeamento precoce ou tardio. No entanto, neste mesmo estudo foi concluído que havia vantagens importantes aos neonatos com o clampeamento tardio, principalmente em melhor peso ao nascer, níveis de hemoglobina adequados e melhores reservas de ferro aos seis meses de idade. Efeitos adversos como a Policitemia não foram observados. O risco de precisar recorrer à fototerapia pós-natal não é considerado elevado desde que ela esteja disponível nos locais da assistência aos recém-nascidos (RN) e os benefícios do clampeamento tardio se sobrepõem a esses $\operatorname{riscos}^{(6)}$.

Sabe-se também que, além dos benefícios hematológicos, já foram estudadas outras vantagens do clampeamento tardio, principalmente em nascimentos prematuros, tais como redução da Hemorragia Intraventricular, redução dos casos de Enterocolite Necrosante e menores índices de Sepse ${ }^{(6-7)}$.

Considerando as evidências mais recentes, o objetivo deste estudo foi analisar a relação entre o tempo de clampeamento do cordão umbilical e incidência de Icterícia Neonatal e níveis de hematócrito em $\mathrm{RN}$ a termo saudáveis em uma maternidade de baixo risco em Curitiba.

\section{Materiais e Métodos}

O projeto foi aprovado pelo Comitê de Pesquisa e Ética do Hospital de Clínicas da Universidade Federal do Paraná, CAAE nº 43241115.0.0000.0096.

O estudo foi realizado na Maternidade Victor Ferreira do Amaral, que faz parte do Complexo

Hospital de Clínicas da Universidade Federal do Paraná e está localizada em Curitiba/PR. A Maternidade é referência no atendimento às gestantes de baixo risco da capital paranaense, e atende em média 300 partos e 900 consultas mensais, pelo Sistema Único de Saúde ${ }^{(8)}$. Trata-se de um estudo transversal quantitativo que avaliou o prontuário de $188 \mathrm{RN}$ a termo saudáveis (37 a 40 semanas), sendo que em 92 destes foram avaliados os níveis de hematócrito, no período de 01 abril de 2015 a 31 de março de 2016.

Para tanto, adotou-se como clampeamento tardio aquele maior ou igual a 60 segundos, e como precoce aquele realizado num tempo inferior a 60 segundos.

Foram incluídos os pares mães-filhos, com gestações saudáveis, do pré-natal de baixo risco; com idade gestacional entre 37 e 40 semanas; com índice de Apgar $\geq 7$ no $1^{\circ}$ minuto; mantidos sobre o ventre da mãe; ambiente aquecido; com idade materna entre 18 e 36 anos e residentes em Curitiba - Estado do Paraná. Os Termos de Consentimento livre e esclarecido (TCLE) foram devidamente assinados. Foram excluídos da análise os RN prematuros ( $<37$ semanas), pós-datismo (>40 semanas), que apresentaram o índice Apgar menor que 7 no $1^{\circ}$ minuto de vida, os nascidos com peso menor de $2500 \mathrm{~g}$, e com quaisquer comorbidades a seguir listadas: Incompatibilidade ABO, Doença Hemorrágica Neonatal, síndromes genéticas e/ou malformações.

As informações utilizadas para geração dos dados foram obtidas através dos prontuários das gestantes e dos $\mathrm{RN}$ até a alta com 48 horas.

Considerou-se $\mathrm{RN}$ com icterícia neonatal quando houve descrição de icterícia no prontuário do RN, no momento da alta hospitalar, com 48 horas de vida, quando a avaliação dos níveis de bilirrubina foi feita por meio de bilirrubinometria transcutânea utilizando o aparelho "bilicheck ${ }^{\circledR}$ ", durante o exame físico. 
Os dados foram agrupados em dois grupos: com e sem icterícia, para avaliar a média do tempo de clampeamento. Avaliou-se o risco relativo de um RN clampeado tardiamente apresentar icterícia, a partir da exposição/não exposição ao fator clampeamento tardio.

Dos 188 pacientes coletados, excluíram-se 12 por não apresentarem relato de icterícia ou não no prontuário e não houve avaliação dos níveis de bilirrubina, e novamente foram divididos em dois grupos: com icterícia e sem icterícia para avaliação de relação com o tempo de clampeamento através de uma regressão logística utilizando o programa STATA 9.1

Para a avaliação dos níveis hematócrito, foram coletadas amostras de $92 \mathrm{RN}$ no momento da alta hospitalar após a $48^{\text {a }}$ hora de vida, quando da realização do "Teste do pezinho", sob a forma de microgotas de sangue obtidas por capilaridade, que posteriormente foram centrifugados e colocados em uma tabela específica para a correta leitura dos hematócritos.

\section{Análise de dados}

Os dados obtidos foram analisados utilizando estatística descritiva e analítica. Os resultados apresentam-se em médias com intervalo de confiança.

O pacote utilizado foi STATA 9.1 e SISA. Foram consideradas associações significativas aquelas que apresentaram $\mathrm{P}<0,05$.

\section{Resultados}

A Tabela 1 descreve os nascimentos em relação à via de parto, sendo que $84,5 \%$ dos RN nasceram de parto transvaginal e $15,5 \%$ de parto cesárea. $54 \%$ dos $\mathrm{RN}$ eram do sexo feminino.

Em relação ao peso dos RN, o Gráfico 1 mostra a

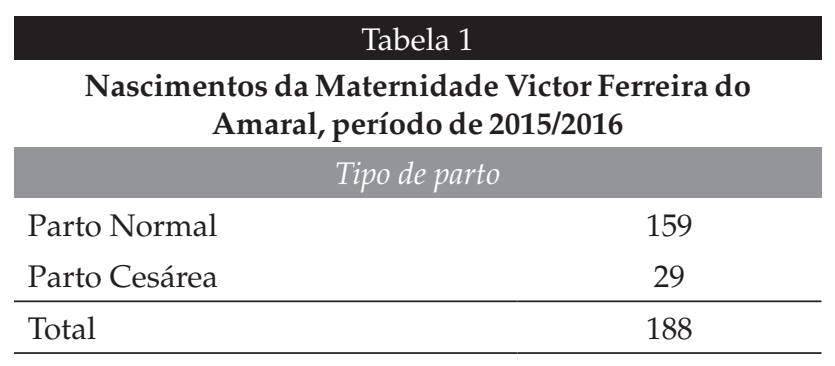

classificação nutricional, no qual $71 \%$ apresentavam-se na faixa de peso de 3000 a $4000 \mathrm{~g} ; 23 \%$ apresentavam-se no intervalo de 2500 a $3000 \mathrm{~g}$ e 5,8\% acima de $4000 \mathrm{~g}$.

Classificação Nutricional

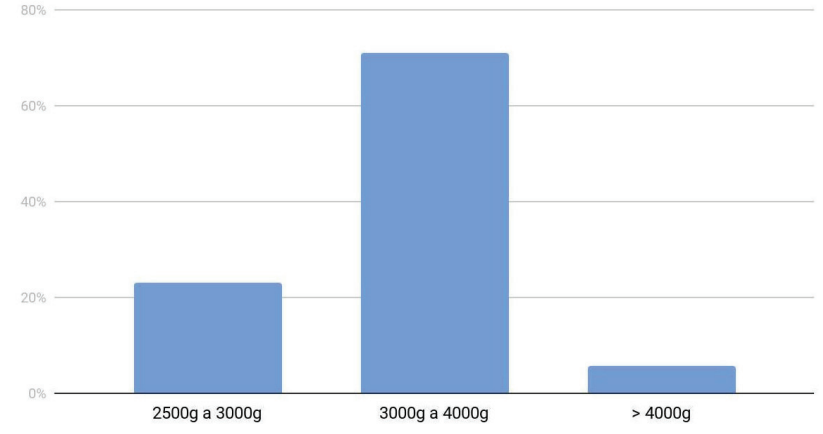

Gráfico 1

Com relação à idade gestacional $4 \%$ tinham 37 semanas ao nascer e $96 \%$ acima de 37 semanas. A Icterícia Neonatal foi registrada em $25 \%$ dos RN. Com relação à média do tempo de clampeamento, no grupo sem icterícia, foi registrado 105 segundos, em comparação com 108 segundos no grupo com icterícia. A associação não foi estatisticamente significante $(p=0,068)$. Com relação ao risco relativo de um RN clampeado tardiamente apresentar icterícia, observou-se um risco 2,18 vezes maior em um $\mathrm{RN}$ clampeado tardiamente do que em um $\mathrm{RN}$ clampeado precocemente $(\mathrm{RR}=2,18$ IC 95\%: 0,15-1,71).

A Tabela 2 apresenta a regressão logística entre o tempo de clampeamento e presença ou não de icterícia em 176 pacientes.

Em relação aos níveis de hematócrito realizados, no grupo de clampeamento tardio o valor médio foi de $58,05 \%$ e destes 09 apresentaram valores $>65,0 \%$. No grupo de clampeamento precoce nenhum valor ultrapassou $65 \%$.

\section{Discussão}

Os resultados nos mostram que o clampeamento tardio não apresenta associação significativa com a Icterícia Neonatal, apesar de demonstrar um risco relativo aumentado. Este maior risco pode ser justificado pela fisiopatologia da própria Icterícia Neonatal: uma

Regressão Logística: tempo de clampeamento do cordão umbilical e icterícia neonatal.

\section{Regressão logística}

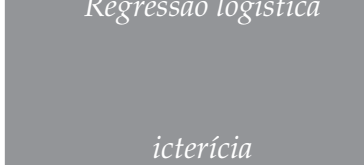

clampeamento
2,833333
1.615637
$N=176$

LR chi2 $=3,99$

Prob $/$ chi $2=0.0459$

Pseudo $R 2=0,0182$ (Intervalo de confiança 95\%] 
maior quantidade de hemoglobina transferida para o RN leva a um aumento fisiológico na formação de bilirrubina indireta por ocasião da degradação da hemoglobina fetal e seu metabolismo, com consequente maior chance de icterícia clínica ${ }^{(9)}$.

O RN apresenta uma condição fisiológica em que produz duas a três vezes mais bilirrubina do que os adultos. Tal característica se deve ao menor tempo de sobrevivência e a maiores níveis de hemoglobina e hemácias neonatais. ${ }^{(9,10)}$. No tocante à Policitemia em neonatos, cabe ressaltar que esta pode ser secundária a produção ativa de novas células sanguíneas vermelhas ou devido a uma transfusão passiva destas células via circulação fetal. Além disso, sabe-se que a Policitemia quando encontrada em RN está também associada ao aumento da viscosidade do sangue, o que gera resistência ao fluxo sanguíneo, principalmente na microcirculação ${ }^{(9-10)}$. Esse aumento na eritropoiese pode ser consequência de estresse ou hipóxia fetal. Tais condições estão relacionadas muitas vezes com restrição no crescimento intrauterino $(\mathrm{RCIU})^{(9-10)}$. Outras causas podem ser citadas, como insuficiência placentária secundária a pré-eclâmpsia, problemas cardíacos maternos, pós-datismo, tabagismo durante a gestação, diabetes gestacional, desordens genéticas ${ }^{(9-10)}$.Nos casos de hipertransfusão e clampeamento tardio, há o aumento do volume de células sanguíneas entregues ao RN. Quando o clampeamento ocorre depois de 3 minutos, o volume sanguíneo pode aumentar em até $30 \%$. Contudo, estudos recentes demonstram que, mesmo em situações em que foi aplicado o clampeamento tardio, os RN não precisaram de tratamento para sintomas como policitemia ${ }^{(11-12)}$

Em relação ao hematócrito, os resultados corroboram a idéia da prevenção da anemia ferropriva nos primeiros seis meses de vida ${ }^{(13)}$. O momento do clampeamento é importante para a reserva de total de ferro no $\mathrm{RN}$, já que um período de tempo maior antes do clampeamento, após ao nascimento, mantém a circulação entre o RN e a placenta através da veia e artérias umbilicais ${ }^{(12-14)}$. A parada na circulação, portanto, terá efeitos profundos no volume sanguíneo e concentração de hemoglobina no $\mathrm{RN}^{(11-15)}$.

Apenas nove RN apresentaram níveis de Hematócrito superior a $65 \%$, sem uma relação clínica, indo na mesma direção de estudos prévios com maiores grupos amostrais, que também indicavam não existir correlação clínica com o maior tempo de clampeamento $^{(1,9,15)}$.

Estudos da década de 1970 indicavam que o RN com maior tempo de clampeamento de cordão apresentavam valores de bilirrubina aumentados além de maior indicação para fototerapia, porém sem consenso sobre esta associação. Algum tempo depois, metanáli- ses concluíram que a indicação para fototerapia é de fato menor nos grupos de clampeamento precoce ${ }^{(11)}$. Desta forma é possível que se adote o tempo de clampeamento preconizado pela OMS de 1 a 3 minutos sem maiores preocupações, se no serviço estiver disponível fototerapia, considerando o risco relativo aumentado para Icterícia Neonatal.

Em metanálises ${ }^{(11,16)}$ os autores sugerem que o tempo de clampeamento de pelo menos 2 minutos, é extremamente benéfico para o $\mathrm{RN}$ até a sua primeira infância. Apesar da associação com a icterícia ser positiva, a condição na maioria das vezes é benigna, e o benefício supera o risco, demonstrado por estudos anteriores ${ }^{(17-18)}$ e em nosso estudo, mesmo em um grupo amostral menor. Estes benefícios, também foram aparentes em relação ao hematócrito, devido ao status hematológico apresentado pelos $\mathrm{RN}$ com clampeamento tardio $(58,05 \%)$, podendo diminuir o risco de anemia entre os lactentes, de acordo com os dados de literatura disponível.

Em nosso trabalho encontramos limitações no que se refere a coleta dos hematócritos de todo nosso grupo amostral devido dificuldades em treinar uma equipe para que executasse os hematócritos, tendo em vista que tal procedimento demanda aprendizagem à parte. Além disso, também temos que ressaltar a dificuldade em aumentar o número da amostra por desencontros no alojamento conjunto com a puérpera e o recém-nascido quando no momento da realização do teste do pezinho e os horários de alta dos RN. Ressalta-se também dificuldades com a equipe de saúde para padronizar a cronometragem do tempo de clampeamento, precisando excluir aquelas que não seguiram o método padronizado. Em relação aos níveis de bilirrubina plasmática, optamos por utilizar o método de bilirrubinometria transcutânea (Bilicheck $®)$, tendo em vista que estudos mostram que este método pode ser utilizado de forma confiável para correlacionar com níveis séricos de bilirrubina ${ }^{(19)}$.

\section{Agradecimentos}

Agradecemos às pacientes gestantes da Maternidade Victor Ferreira do Amaral pelo consentimento com a coleta do hematócrito, para que essa pesquisa fosse possível.

Às profissionais da enfermagem pela ajuda e pelo empenho na anotação de diversos dados dos recém-nascidos, incluindo o tempo de clampeamento do cordão.

À Maternidade Vitor Ferreira do Amaral pelo acolhimento durante o desenvolvimento desta pesquisa, bem como a participação da equipe de neonatologistas. 


\section{Referências}

1. Ceriani Cernadas JM. Timing of umbilical cord clamping of term infants. Arch Argent Pediatr. 2017; 115(2):188-94.

2. Organisation Mondiale de la Santé. Clampage du cordon pour la prévention de l'anémie ferriprive chez les nourrissons: moment optimal. Dernière mise à jour:

5 février 2019. [Internet]. Bibliothèque électronique de données factuelles pour les interventions nutritionnelles (eLENA). [cité 2019 Mar 12]. Disponible en: https://www.who.int/elena/ titles/cord_clamping/fr/

3. World Health Organization. Guideline: delayed umbilical cord clamping for improved maternal and infant health and nutrition outcomes. [Internet]. Geneva: World Health Organization; 2014. 28p. [cited 2019 Mar 12]. Available from: https://apps.who. int/iris/bitstream/handle/10665/148793/9789241508209_eng. pdf?ua $=1$

4. Chaparro CM. Timing of umbilical cord clamping: effect on iron endowment of the newborn and later iron status. Nutr Rev. 2011; 69 (Suppl 1):S30-6.

5. Rincón D, Foguet A, Rojas M, Segarra E, Sacristán E, Teixidor $\mathrm{R}$, et al.Tiempo de pinzamiento del cordón umbilical y complicaciones neonatales, un estudio prospectivo. An Pediatr (Barc). 2014;81(3):142-8.

6. Mercer JS, Erickson-Owens DA, Collins J, Barcelos MO, Parker $\mathrm{AB}$, Padbury JF. Effects of delayed cord clamping on residual placental blood volume, hemoglobin and bilirubin levels in term infants: a randomized controlled trial. J Perinatol. 2017;37(3):260-4.

7. McDonald SJ, Middleton P, Dowswell T, Morris PS. Effect of timing of umbilical cord clamping of term infants on maternal and neonatal outcomes. Cochrane Database Syst Rev. 2013; (7):CD004074

8. Brasil. Ministério da Educação. EBSERH - Hospitais Universitários Federais. Complexo Hospital de Clínicas. [Internet]. MVFA - Maternidade Victor Ferreira do Amaral. [citado 2019 Mar 12]. Disponível em: http://www2.ebserh.gov.br/web/ chc-ufpr/maternidade-victor-do-amaral

9. Kandasamy, J. Polycythemia of the newborn. Medscape. [Internet]. Updated: Dec 22, 2017. [cited 2019 Mar 10]. Available from: https://emedicine.medscape.com/article/976319-overview
10. Mondadori C. Hiperbilirrubinemia do RN a termo e a experiência materna mediante a fototerapia. Rev Uniplac.[Internet]. 2018 [citado 2019 Mar 10]: 6(1). Disponível em: http:/ / revista. uniplac.net/ojs/index.php/uniplac/article/view/3655

11. Hutton EK, Hassan ES. Late vs early clamping of the umbilical cord in full-term neonates: systematic review and meta-analysis of controlled trials. JAMA. 2007;297(11):1241-52.

12. Chien PC, Yang CC, Gau ML, Liu CY, Lee TY. [The Impact of Late Umbilical Cord Clamping on Neonatal Jaundice and Postpartum Hemorrhage: A Randomized Controlled Trail]. Hu Li Za Zhi. 2015;62(4):41-53.

13. Di Sarli YO. Associação entre tempo de clampeamento do cordão umbilical e icterícia neonatal precoce em recém-nascidos a termo. Dissertação (Mestrado). São Paulo: Universidade Santo Amaro; 2018.

14. Linderkamp O, Nelle M, Kraus M, Zilow EP. The effect of early and late cord-clamping on blood viscosity and other hemorheological parameters in full-term neonates. Acta Paediatr. 1992; 81(10):745-50.

15. Andersson O, Lindquist B, Lindgren M, Stjernqvist K, Domellöf M, Hellström-Westas L. Effect of delayed cord clamping on neurodevelopment at 4 years of age: a randomized clinical trial. JAMA Pediatr. 2015; 169(7):631-8.

16. Rabe H, Diaz-Rossello JL, Duley L, Dowswell T. Effect of timing of umbilical cord clamping and other strategies to influence placental transfusion at preterm birth on maternal and infant outcomes. Cochrane Database Syst Rev. 2012; (8):CD003248.

17. Tarnow-Mordi W, Morris J, Kirby A, Robledo K, Askie L, Brown $\mathrm{R}$, et al. Delayed versus Immediate Cord Clamping in Preterm Infants. N Engl J Med. 2017; 377(25):2445-55.

18. Jahazi A, Kordi M, Mirbehbahani NB, Mazloom SR. The effect of early and late umbilical cord clamping on neonatal hematocrit. J Perinatol. 2008; 28(8):523-5.

19. Alsaedi SA. Transcutaneous bilirubin measurements can be used to measure bilirubin levels during phototherapy. Int J Pediatr.2016; 29(16):2635-9.

Trabalho recebido: $24 / 03 / 2019$

Trabalho aprovado: 11/07/2019

Trabalho publicado: 12/07/2019 